\title{
A Systematic Review for Clinical Assessment of Syncope Pediatric Patients

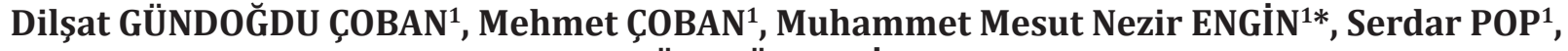 Öner ÖZDEMIR ${ }^{2}$
}

\author{
${ }^{1}$ Sakarya University Training and Research Hospital, Department of Pediatrics, Turkey. \\ ${ }^{2}$ Sakarya University Training and Research Hospital, Division of Pediatric Immunology and Allergy, Turkey. \\ *Corresponding Author: Muhammet Mesut Nezir ENGIN, Sakarya University Training and Research Hospital, \\ Department of Pediatrics, Turkey.
}

Abstract

Definition: Syncope is a common clinical problem of pediatric emergency department that generates a great amount of anxiety among patients, families and even physicians. It is a sudden, brief loss of consciousness associated with loss of postural tone from which recovery is spontaneous. The etiology of syncope in children is most often benign but it can also occur as the result of more serious disease with the potential for sudden death (especially cardiac reasons). The differential diagnosis between seizures and syncope is very important. The classification of syncope is divided into three major categories as neurally mediated syncope, cardiovascular syncope, and non-cardiovascular syncope.

Clinical Features: The major difficulty for the assessment of children with syncope is that most of them are asymptomatic at the time of presentation, therefore taking a detailed history and performing a good physical examination are very important to rule out the cardiac reasons and avoid the misdiagnosis of epilepsy all patients. Excluding the life-threatening reasons, and management of the reason can decrease patient's and family's anxiety as well as improve quality of life.

Conclusion: The aims of the review is to conduct a systematic review of pediatric syncope in terms of etiologies to identify the most common causes. Detailed evaluation should be considered for patients who have syncope during exercise or who have a family history of syncope, sudden death, myocardial disease or arrhythmias. Additional testing will have a low yield and not be cost-effective for patients without risks of cardiac with a normal physical examination.

Keywords: Congenital Heart Diseases, Epilepsy, Syncope, Loss of Consciousness, Vasovagal

\section{INTRODUCTION}

Syncope is a common pediatric complaint with sudden, brief and transient loss of consciousness associated with loss of postural tone with a spontaneous recovery. It is self-limited loss of consciousness and postural tone followed by spontaneous and complete recovery without any neurological sequelae (1). There is also presyncope which is the feeling that one is about to pass out but remains conscious with a transient loss of postural tone (2).

Approximately $\% 15$ of children experience a syncopal episode prior to the end of adolescence (3). The reasons of syncope in the pediatric patients range from vasovagal to cardiac to seizure disorders (4). It can be classified according to the underlying cause: neurogenic (eg, vasovagal, situational, and orthostatic syndromes), cardiac, metabolic, or a condition that mimics syncope. Based upon case series of pediatric patients evaluated in the emergency department, vasovagal type comprises most of cases (3-6). Zavala et al., In a study conducted on 3700 patients with pediatric syncope in 2020, found the most common etiological causes as vasovagal syncope (52.2\%), idiopathic (18.3\%) and postural orthostatic tachycardia syndrome (13\%). In addition, the cardiac 
causes most worried by the families were found at a rate of 4\% (7). Driscoll and colleagues reported an overall incidence of 125 of 100,000 (0.125\%) for pediatric patients seeking medical attention (8).

The other name of vasovagal syncope is neurally mediated syncope (NMS). NMS is characterized by systemic hypotension resulting in inadequate cerebral blood flow from a reflex-mediated combination of inappropriate vasodilatation and bradycardia (9). The diagnostic criteria for vasovagal syncope include the absence of any other reason or etiology, a positive response to positional change when symptoms start with a vasovagal reaction (hypotension and/or bradycardia) (10). Most of them do not require any medications and do well with lifestyle modifications, such as increased water intake $(9,11)$.

Neurologic etiologies, such as seizures or migraine headaches, are present in up to 20 percent of patients. There are relatively rare (1 to 2 percent of children) reasons but occur frequently enough to require diagnostic importance. These lifethreatening conditions could be metabolic reasons (eg. Hypoglcemia), anaphylaxis, cardiac arrhythmia (tachyarrhythmias, long QT syndrome, Brugada syndrome, bradycardia), congenital cardiac diseases (eg. Fallot tetralogy, dilated cardiomyopathy, aortic stenosis, hypertrophic cardiomyopathy) or acute myocarditis (12-13). There are also some conditions that may mimic syncope include the seizures, hyperventilation, hysteria/conversion disorder, migraine syndromes $(3,6)$. In a study of McHarg et al. reported that only $6 \%$ of 108 pediatric patients had cardiac-originated syncope (14). Ritter et al. presented a study of 480 pediatric syncope patients, electrocardiograms (ECG) were performed in 449/480 (94\%) of patients and were abnormal in $15 \%$ and echocardiograms were performed in 322 (67\%) patients and were abnormal in $12 \%$. In this study noncardiac causes were found in 95\%, including 71\% vasovagal syncope (13).

In most cases, the underlying cause of syncope in the pediatric patient can be determined by obtaining thorough the history and physical examination (2-6). Paying attention to cardiac, neurological, and psychological medical history and performing a physical examination can rule out more rare and important causes of syncope $(2-7,12)$.

\section{Evaluation of Patient}

A standardized approach to the child or adolescent presenting with syncope is needed. The American
Heart Association, the American College of Cardiology, and the European Society of Cardiology have created guidelines for the evaluation of syncope. These guidelines recommend that the essential components of evaluation of children who present with syncope includes detailed medical and family history, physical examination, and electrocardiogram (ECG) (15). The goal of the evaluation of a syncope patient presenting with loss of consciousness is to identify and distinguished from all life-threatening conditions $(7,12)$. There are many ways to classify syncope, but the simplest is to divide the causes of syncope into three groups: neurogenic, cardiac and noncardiac reasons (3-4-6-7-11).

After the patient is stabilized, a detailed history of the event and a thorough examination are required to determine the etiological cause. Preceding events or precipitating factors must be asked about triggering factors like physical effort (it is concerning for a cardiac reason, but vasovagal syncope is also common during physical exercise), postural changes, emotional stress, comorbidities (eg. Metabolic diseases, DM), medications (eg.insulin), substance abuse, painful stimulants, hot weather, hunger (hypoglycemia), lack of sleep, prolonged standing, micturition, or defecation (6).

A detailed family history must be taken. First or second-degree relatives for familial cardiomyopathy, early cardiac death ( $<50$ years), known arrhythmias (Brugada Syndrome, Long QT Syndrome etc.) and sudden unexplained deaths increases the risk of cardiac reasons. It could suggest a genetically inherited cause of cardiac syncope $(6,8,16,17)$. Cardiac causes represent the minority of syncope cases, they should not be missed.

The first etiological reason to be evaluated in syncope is cardiac syncope since it may require urgent intervention. In most children, cardiac syncope can be evaluated in the differential diagnosis with a careful history taking (detailed family medical history), physical examination, vital signs and ECG. History and physical examination together may be $95 \%$ sensitive for a cardiac etiology (18).

A description of the event must be asked to the child and witnesses should include; the child's symptoms prior to the onset of syncope and the syncope moment. Patients with vasovagal syncope frequently report prodromal symptoms that include light-headedness, dizziness, sweating, nausea, and visual changes. They may experience fatigue after the syncope. 
In a previous study involving pediatric patients, McHarg and colleagues found no clinical or historical features to reliably distinguish cardiac causes of syncope from noncardiac causes such as neurally mediated syncope (14).

Syncope followed by abnormal motor activity (ie, tonic clonic movement or posturing) can occur during the event or at the end of a syncope or and most often correlates with seizures (19). It is important to clinically differentiate between an epileptic and a syncopal attack. Some of the seizure types such (myoclonus and drop-attacks) may be confused with syncope, however, the way of the event occurred and associated neurological impairment help distinguish them from a syncopal attack. In syncope duration of such activity is usually short, and recovery is rapid, consciousness returns within minutes of lying down and unconsciousness for more than $5 \mathrm{~min}$ is rare and unusual (19-20).

Vital signs should be noted during and after the syncope if possible. First line laboratory tests (complete blood count, glucose, electrolytes, urine test, arterial blood gas and serum toxin scan) and ECG are usually enough, and there is no need for further investigations for the diagnosis of most patients. The obtained results should be used as a guide for further diagnostic studies in patients with suspected diagnosis (21-22).

Metabolic reasons and medications can cause syncope. Symptoms such as dizziness, lightheadedness, pallor and sweating are observed. The main metabolic disorders thatlead to syncopeare electrolyte imbalance (hypocalcemia, hypomagnesemia) hypoglycemia, hyperammonemia, medications (most commonly macrolides and antihistamines), intoxication, anorexia nervosa, hyperventilation and hypoxia. Duration of consciousness is longer, and symptoms may persist until the underlying cause is treated $(2,4,14,23-26)$.

Detailed cardiovascular examination is required for patients who have syncope during exercise $(15,21,22)$. ECG is considered a standard part of the syncope work and must be taken in all patients with syncope. Cardiac rhythm, corrected QT interval, and heart rate should be evaluated carefully in each patient. If the ECG is normal at the initial evaluation, patients who may require a more extensive cardiac evaluation (i.e., additional ECG monitoring, echocardiography, or a stress test) if there is risk for cardiac reasons $(21,22)$ (23). Further evaluation may be needed in selected cases; include echocardiography, Holter-monitoring and rarely, cardiac catheterization $(14,23)$. Echocardiography should be performed in selected patients in suspicion of congenital cardiac disease but the diagnostic value of echocardiography is $0.5-10 \%$ (9-25). It was shown that echocardiography (ECHO) does not help if you do not have any suspicious findings in the history and physical examination. The diagnostic value of the Holter test ranges between 0.4 and $19 \%$ (27).

Electroencephalography (EEG) and neuroimaging studies (computer tomography) may be needed in children with neurological findings or if there is a strong suspicion of seizure despite syncope. EEG may help in distinguishing a convulsive syncope from a true epileptic seizure. EEG investigation is performed commonly in the evaluation of patients with syncope. The diagnostic value of this test is $1.5 \%$, which is very low (28).

\section{MANAGEMENT}

The management of pediatric syncope requires recognition of the main reason and treatment of the underlying cause. If syncope is not due to a cardiac cause after complete recovery, most of children can be followed as outpatients. The initial management is to stabilize the patient, focusing on the airway, breathing, and circulation (ABC). Intravenous access, oxygen administration, and cardiac monitoring should be performed. The patient should be evaluated for metabolic causes and should be treated for the specific cause.

Patients with recurrent vasovagal (neurocardiac) syncope that does not respond to empiric treatment with increased fluid and salt intake may warrant an elective pediatric cardiology consultation $(9,11,29)$. The assumption of a supine posture, elevation of the legs and lowering the head, leg crossing and tensing of the abdominal muscles decrease pooling of venous blood in the lower limbs and abdomen and/or increase peripheral arterial resistance $(14,22,23,29)$. Patients should keep away from personal and environmental triggering factors $(25,29)$. If severe syncope attacks persist, medical treatment may be started after cardiology consultation.

Patients with a suspicion of cardiac syncope based upon history, physical examination or ECG should be consultation or referral to a pediatric cardiologist 
before discharge the hospital (6-8,29). The main drugs used for pharmacological treatment are betablockers, scopolamine, fludrocortisone, theophylline, ephedrine, midodrine, clonidine, and serotonin reuptake inhibitors. Today, it is accepted that beta-blockers are a first-line safe drug that can be used for this purpose (20).

In summary, the vitals of the patient who applied to the Pediatric Emergency Service with syncope should be evaluated first and ECG should be taken. If there is a disorder in these values or in the general condition of the patient, necessary intervention should be made. Afterwards, after the general condition is good and the vitals are stable, a detailed medical history should be questioned and a detailed physical examination should be performed to investigate the etiological cause of syncope. Complete blood count, glucose and electrolyte levels should be examined. According to the vital signs, ECG, medical history, physical examination and test results, the differential diagnosis of syncope should be made, and further investigation, follow-up and treatment should be planned for the etiological cause.

\section{ConCLUSIONS}

Syncope should be differentiated from cardiac reasons, epileptic seizures and psychogenic causes. It frequently requires medical examination or admission to pediatric departments and majority of cases does not subtend serious diseases but nevertheless, it always requires a complete medical evaluation in order to exclude underlying pathology. It is very important to differentiate cardiac and non-cardiac (metabolic, neurologic, drug induced) reasons of syncope. Therefore, a detailed history should be taken, then physical examination should be performed, and routine laboratory tests and ECG must be conducted in each child with syncope. Other investigations are required if clinical data and the ECG do not provide an etiological explanation of the episode in any clinical suspicion of life-threatening reasons.

\section{REFERENCES}

[1] Kapoor WN. Syncope. N Engl J Med 2000; 343:1856.

[2] Fischer JW, Cho CS. Pediatric syncope: cases from the emergency department. Emerg Med Clin North Am. 2010;28:501-16.

[3] Massin MM, Bourguignont A, Coremans C, et al. Syncope in pediatric patients presenting to an emergency department. J Pediatr 2004; 145:223.

[4] Zhang O Du J Wang C, et al. The diagnostic protocol in children and adolescents with Syncope: a multi-centre prospective study. Acta Paediatr. 2009;98:879-884.

[5] Bo I, Carano N, Agnetti A, et al. Syncope in children and adolescents: a two-year experience at the Department of Paediatrics in Parma. Acta Biomed 2009; 80:36.

[6] Friedman KG, Alexander ME. Chest pain and syncope in children: a practical appro-ach to the diagnosis of cardiac disease. J Pediatr 2013; 163:896.

[7] Zavala R, Metais B, Tuckfield L, DelVecchio M, Aronoff S. Pediatric Syncope: A Systematic Review. Pediatr Emerg Care. 2020;36(9):442-445.

[8] Driscoll DJ, Glicklich LB, Gallen WJ. Chest pain in children: a prospective study. Pediatrics. 1976;57:648-51.

[9] Johnsrude CL. Current approach to pediatric syncope. Pediatr Cardiol 2000;21:522-53.

[10] Tao C, Tang C, Chen S, et al. Autonomic Nervous Function in Vasovagal Syncope of Children and Adolescents. Neuroscience Bulletin. 2019;35: 937-940.

[11] Goble MM, Benitez C, Baumgardner M, Fenske K. ED management of pediatric syncope: searching for a rationale. Am J Emerg Med 2008; 26:66.

[12] Hurst D, Hirsh DA, Oster ME, et al. Syncope in the Pediatric Emergency Department - Can We Predict Cardiac Disease Based on History Alone? J Emerg Med 2015; 49:1.

[13] Ritter S, Tani LY, Etheridge SP, et al. What is the yield of screening echocardiography in pediatric syncope? Pediatrics 2000; 105:E58.

[14] McHarg M, Shinnar S, Rascoff H, et al. Syncope in childhood. Pediatr Cardiol. 1997;18:367-371.

[15] American College of Emergency Physicians. Clinical policy: critical issues in the evaluation and management of patients presenting with syncope. Ann Emerg Med 2001; 37:771.

[16] Gillette PC, Garson A Jr. Sudden cardiac death in the pediatric population. Circulation 1992; 85:I64. 
[17] Temizkan R, Engin MMN, Kaya F, Çolak M, Kocabay K. Acute myocarditis that confounded by acute myocardial infarction in adolescent patients who applied Pediatric emergency room with chest pain. Fam Pract Palliat Care. 2017; 2(2): 18-21.

[18] Crompton DE, Berkovic SF. The borderland of epilepsy: clinical and molecular features of phenomena that mimic epileptic seizures. Lancet Neurol. 2009;8:370-81.

[19] Benbadis SR, Chichkova R. Psychogenic pseudosyncope: an underestimated and provable diagnosis. Epilepsy Behav. 2006;9: 106-10.

[20] Grubb BP, Gerard G, Wolfe DA, et al. Syncope and seizures of psychogenic origin: identification with head-upright tilt table testing. Clin Cardiol. 1992;15:839-42.

[21] Tanel RE, Walsh EP. Syncope in the pediatric patient. Cardiol Clin 1997; 15:277.

[22] Prodinger RJ, Reisdorff EJ. Syncope in children. Emerg Med Clin North Am 1998; 16:617.

[23] Matoth I, Taustein I, Kay BS, Shapira YA (2002) Overuse of EEG in the evaluation of common neurologic conditions. Pediatr Neurol 27:378-383.

[24] Van Dijk N, Quartieri F, Blanc JJ, Garcia-Civera R, Brignole M, Moya A, PC-Trial Investigators et al
(2006) Effectiveness of physical counterpressure maneuvers in preventing vasovagal syncope: the Physical Counterpressure Manoeuvres Trial (PCTrial). J Am Coll Cardiol 48:1652-1657.

[25] Ikiz MA, Cetin II, Ekici F, Güven A, Değerliyurt A, Köse G (2014) Pediatric syncope: is detailed medical history the key point for differential diagnosis? Pediatr Emerg Care 30:331-334.

[26] MacNeill EC, Vashist S (2013) Approach to syncope and altered mental status. Pediatr Clin North Am 60:1083-1106.

[27] Hegazy RA, Lotfy WN (2007) The value of Holter monitoring in the assessment of pediatric patients. Indian Pacing Electrophysiol J 7:204-214.

[28] Matoth I, Taustein I, Kay BS, Shapira YA (2002) Overuse of EEG in the evaluation of common neurologic conditions. Pediatr Neurol 27:378-383.

[29] Bayram AK, Pamukcu O, Per H. Current approaches to the clinical assessment of syncope in pediatric population. Childs Nerv Syst. 2016;32(3):427-36.

Citation: Dilşat GÜNDOĞDU ÇOBAN, Mehmet ÇOBAN, Muhammet Mesut Nezir ENGíN, Serdar POP, Öner ÖZDEMIR. A Systematic Review for Clinical Assessment of Syncope Pediatric Patients. Archives of Emergency Medicine and Intensive Care. 2021; 4(1): 09-13. DOI: https://doi.org/10.22259/2638-5007.0401002

Copyright: (c) 2021 Dilşat GÜNDOĞDU ÇOBAN, Mehmet ÇOBAN, Muhammet Mesut Nezir ENGíN, Serdar POP, Öner ÖZDEMIR. This is an open access article distributed under the Creative Commons Attribution License, which permits unrestricted use, distribution, and reproduction in any medium, provided the original work is properly cited. 